Technical Progress Report

15 May '91-14 April '92

\title{
KNOT INVARIANTS AND THE THERMODYNAMICS OF LATTICE GAS AUTOMATA \\ DE FG03-91ER-14176
}

\author{
Principal Investigator: \\ David A. Meyer \\ Institute for Pure and Applied Physical Science \\ University of California/San Diego \\ La Jolla, CA 920s'3-0075
}

DOE/ER/14176-Tl.

\section{Year 1: progress to date}

The goal of this project is to build on the understanding of the connections between knot invariants, exactly solvable statistical mechanics models and discrete dynamical systems that we have gained in earlier work $[1,2]$, toward an answer to the question of how early and robust thermodynamic behavior appears in lattice gas automata. In [3] we showed that a specific reversible cellular automaton model with a conserved quantity is equivalent, in the sense that the ensemble of $1+1$ dimensional spacetime evolutions is the same as the equilibrium ensemble of 2 dimensional configurations, to a solvable statistical mechanics model. This model, the asymmetric six vertex model, is obtainable as a critical limit of the eight vertex model, which is also solvable. The dominant characteristic of criticality is scale or conformal invariance, which means that probability distributions of states are invariant and correlation functions transform covariantly under conformal transformations. Thus, if our cellular automaton were really at a critical point, i.e., $N \rightarrow \infty$ as well as $T^{\prime}=T_{\text {critical }}$, the local distribution of states would be that of the thermodynamic limit, a result consistent with the canonical distribution observed in simulations. For finite $N$ this will be moderated by finite-size effects which round off the critical singularity.

Having applied these ideas successfully to the simple case of a reversible cellular automaton, we moved on to the more interesting case of lattice gases. Since our approach equates the spacetime evolution of a dynamical system with an equibrium configuration of a statistical mechanics model in one higher dimension, a model of 't Hooft for two dimensional spacetime with discrete local coordinate invariance was a natural inspiration. In [4] we detail the construction of a family of 1 dimensional lattice gas automata based on this model. In an effort to clarify which features of the lattice gas automata are the consequences of which constraints, we proceed systematically: first setting the kinematics of the model, then exploring the effect of 


\section{DISCLAIMER}

This report was prepared as an account of work sponsored by an agency of the United States Government. Neither the United States Government nor any agency thereof, nor any of their employees, makes any warranty, express or implied, or assumes any legal liability or responsibility for the accuracy, completeness, or usefulness of any information, apparatus, product, or process disclosed, or represents that its use would not infringe privately owned rights. Reference herein to any specific commercial product, process, or service by trade name, trademark, manufacturer, or otherwise does not necessarily constitute or imply its endorsement, recommendation, or favoriay by the United States Government or any agency thereof. The views and opinions of authors expressed herein do not necessarily state or reflect those of the United States Government or any agency thereof. 


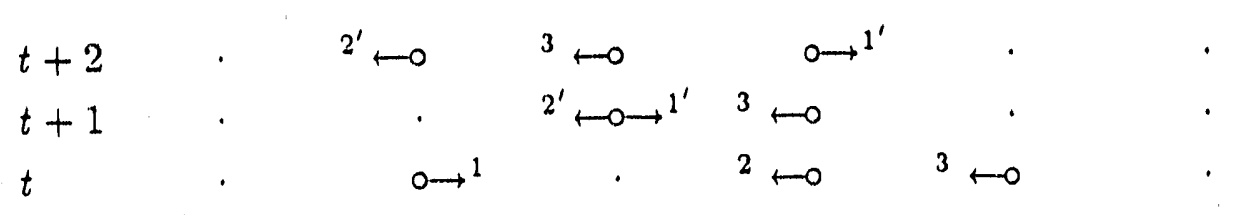

Figure 1.

requiring that the dynamics be local, and finally constructing the possible dynamics consistent with the imposed constraints.

The most interesting of these three steps is the second. By local dynamics we mean that two particles interact only when they coincide in both space and time. The simplest consequence of this requirement is illustrated in Figure 1. There particles 1 and 2 interact at time $t+1$, but particles $1^{\prime}$ and 3 move past each other without interacting because their spatial coordinates always differ by an odd number, precluding coincidence. This apparently trivial observation is at the origin of the "spurious" conserved quantities often found in lattice gas models: here the gas decouples into two noninteracting gases, one occupying the even nodes of the lattice and the other the odd. In [3] we used the conserved quantity of the cellular automaton to define the weights of the equivalent statistical mechanics model. Here, rather than follow the usual approach to dealing with a spurious conserved quantity (modifying the model in an attempt to break the unwanted conservation law) we use it to effect a radical transformation of the model to one which is again equivalent to a 2 dimensional statistical mechanics model. The transformation is described in [4]; to our knowledge this is the first time such an approach has been taken.

The conclusion is that the model lies on a first order critical line between disordered and totally ordered regimes of the six vertex model. We observe that this result applies to ensembles of systems, in the thermodynamic limit. That the correlation length vanishes, for example, does not mean that there is no correlation between states of the probabilistic lattice gas automaton at successive time steps. Nor would a nonvanishing correlation length mean that there were necessarily spacelike correlations in a given initial state. Instead, the interpretation is that deep in the interior of a system, far from the boundary conditions and thus/or subject to essentially random boundary conditions, a domain of sufficiently large size is in thermodynamic equilibrium. Thus our results provide evidence for the first step in the derivation of the macroscopic equations of motion-the assumption of local thermodynamic equilibrium, as well as demonstrating the efficacy of constructing and analyzing lattice gas automata according to (spacetime) symmetry principles.

\section{Year 1: current developments}

In 't Hooft's original model the particle trajectories were to be interpreted as defect lines in a (spacetime) lattice which developed curvature at the crossing (interac- 
tion) points. Our immediate concern then, at least for this model, is to determine the consequences for this interpretation of our transformation to a solvable statistical mechanics model and the conclusions about its thermodynamics that we thereby draw. Our results should be compared to a model where the energy of a configuration depends on the curvature as it does both in models for spacetime and models for crystalline materials. Of course, this also raises the general question of what energies/weights might be appropriate when such a simple lattice structure is not available. For an interacting particle model of a generic dynamical system the weakest information to which one would have access is the causal ordering of the interactions; our work to this point suggests addressing the question of whether at least some such models could be mapped into a statistical mechanics framework; though one could not expect the resulting model to be solvable, some understanding of the dynamical system might be gained nevertheless.

It was our proposed intention to investigate other 1 dimensional systems, like the cellular automaton model for Burger's equation and the thermodynamic formalism for iterated maps, before attacking the much more difficult higher dimensional case. One of our new and most exciting ideas is based on the remarkable fact that there are 1 dimensional cellular automati which are universal Turing machines. This means that they can simulate any deterministic computation including, for example, the evolution of a higher dimensional lattice gas, albeit slowly. Part of the description of a computation of such a Turing machine is a string of letters representing successive states of the machine. This is also reminiscent of the symbolic dynamics approach to dynamical systems. We would like to treat a string arising in either context as a configuration of a 1 dimensional statistical mechanics model. The appropriate energy/weight would depend on exactly which model is being considered. While similar ideas have been considered for deterministic finite automata (a weaker class of machines), we can find no reference to work on the universal Turing rnachine, but believe it is worth exploring.

\section{Year 2}

\section{Research planned}

We intend to focus the bulk of our research efforts in the directions described in the previous section:

Studying the relation between our lattice gas analysis of 't Hooft's model and the curvature interpretation.

- Investigating the possibility of mapping generic interacting particle systems to statistical mechanics models from weaker information than a square lattice of interactions.

Applying the framework developed thus far to other one dimensional models, 
such as the cellular automaton for Burger's equation.

- Extending this approach to more powerful models such as universal Turing machines and symbolic dynamical systems.

In addition, we would like to begin investigating the higher dimensional case directly, looking for solutions to the Zamolodchikov tetrahedron equation described in our original proposal. We expect this to be a very difficult problem, but with the aid of symbolic manipulation programs such as Mathematica, perhaps not intractable.

Other research support

We obtained continued funding by INCOR for partial support of collaboration with Brosl Hasslacher at Los Alamos National Laboratory. At the suggestion of Lu Sham, the new director of IPAPS, we are exploring the possibility of moving to the Program in Geometry and Physics, also at UCSD. This would promote interaction with Michael Freedman and Hans Wenzl in the mathematics department, both of whom have provided helpful discussions about the Yang-Baxter equation and will be valuable resources when we begin investigation of the tetrahedron equation.

\section{Budget}

There have been no major changes in the budget proposed, though a SPARC workstation has not yet been purchased and a change in the status of the Principal Investigator to Assistant Research Mathematician is expected this quarter.

\section{Publications}

[1] D. A. Meyer, "State models for link invariants from the classical Lie groups," to appear in the Proceedings of the International Conference on Knot 'Theory and Related Topics, Osalia, Japan, 15-19 August 1990 (Berlin: W. de Gruyter).

[2] B. Hasslacher and D. A. Meyer, "Knot invariants and cellular automata," Physica D45 (1990) 328-344.

"[3] B. Hasslacher and D. A. Meyer, "Knots, criticality and thermodynamics in discrete dyn mical systems," submitted to Phys. Lett. A.

*[4] B. Hasslacher and D. A. Meyer, "Lattice gases and exactly solvable models," submitted to J. Stat. Phys.

* indicates that a copy of the preprint is enclosed. 

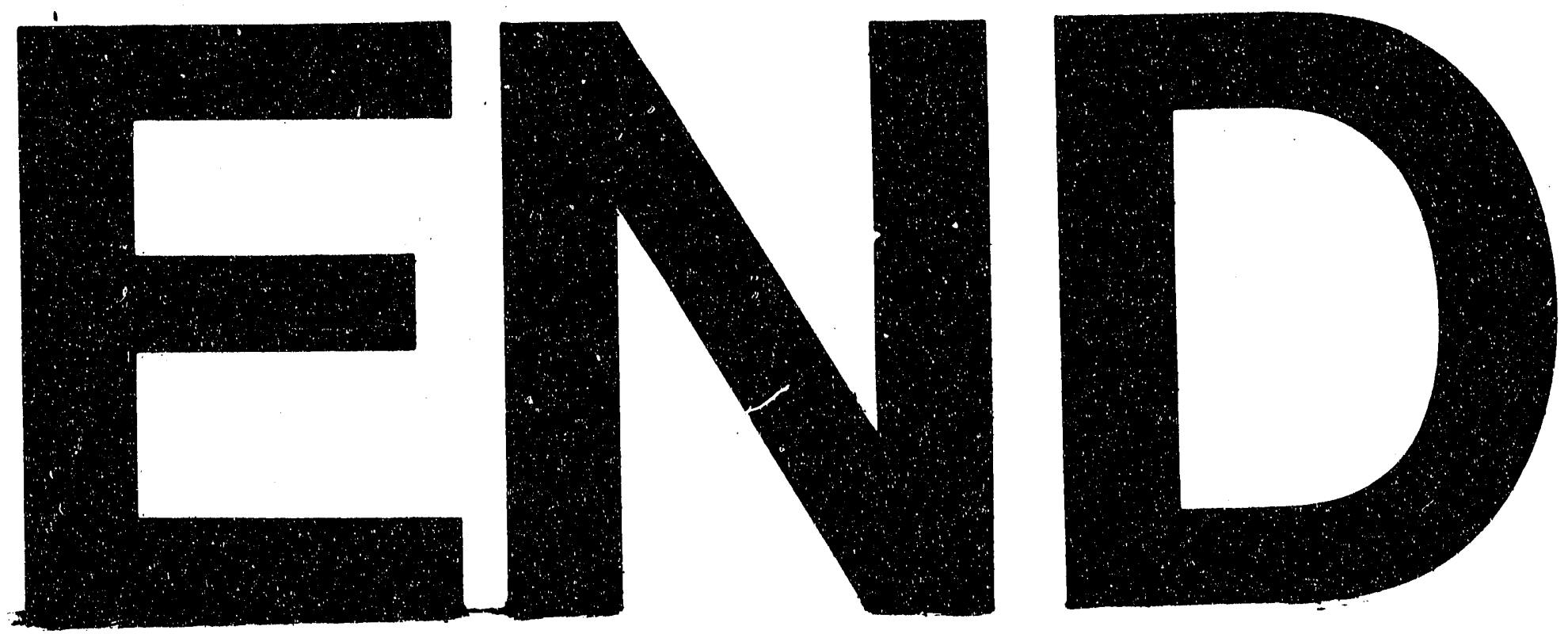

(2)
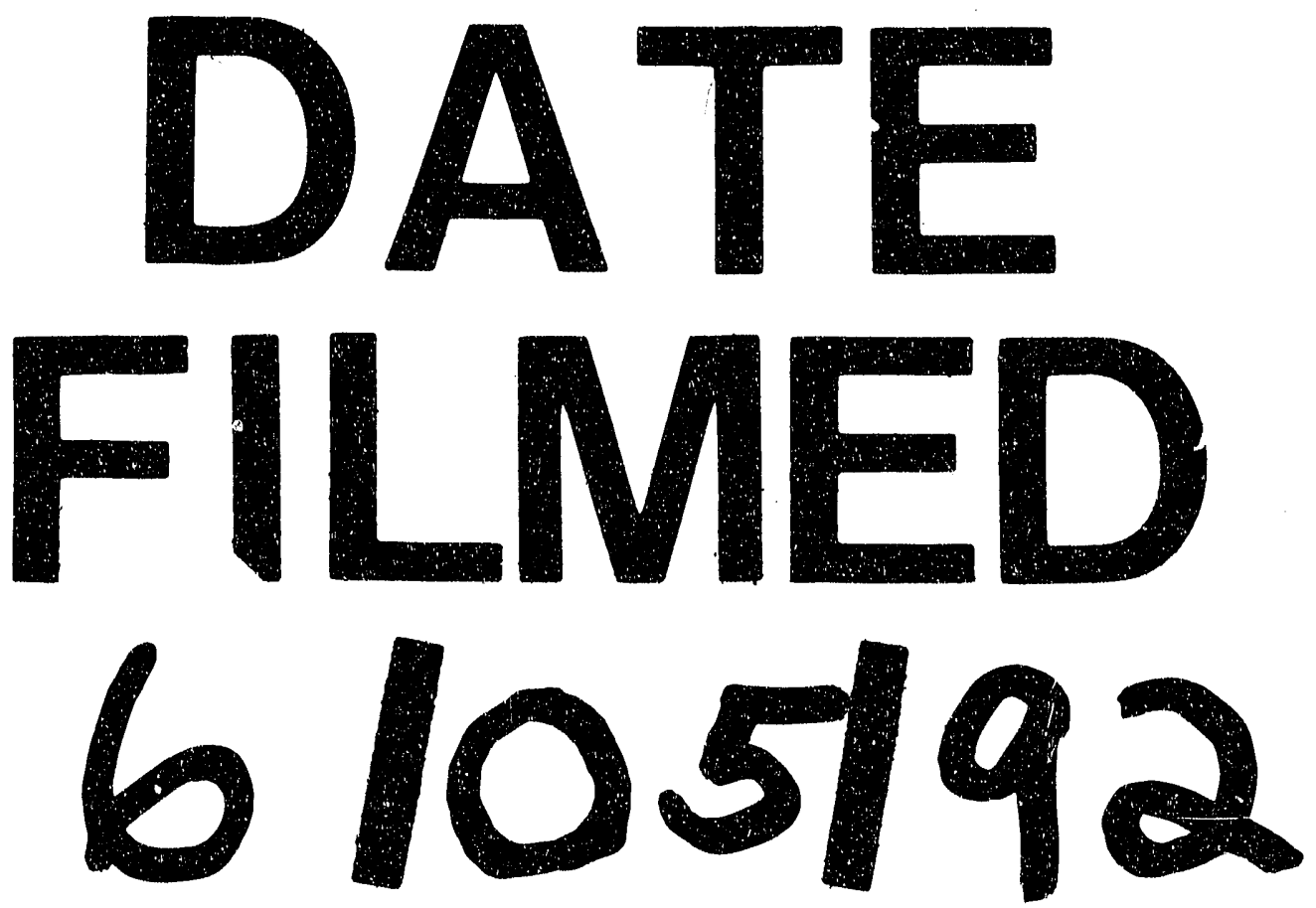


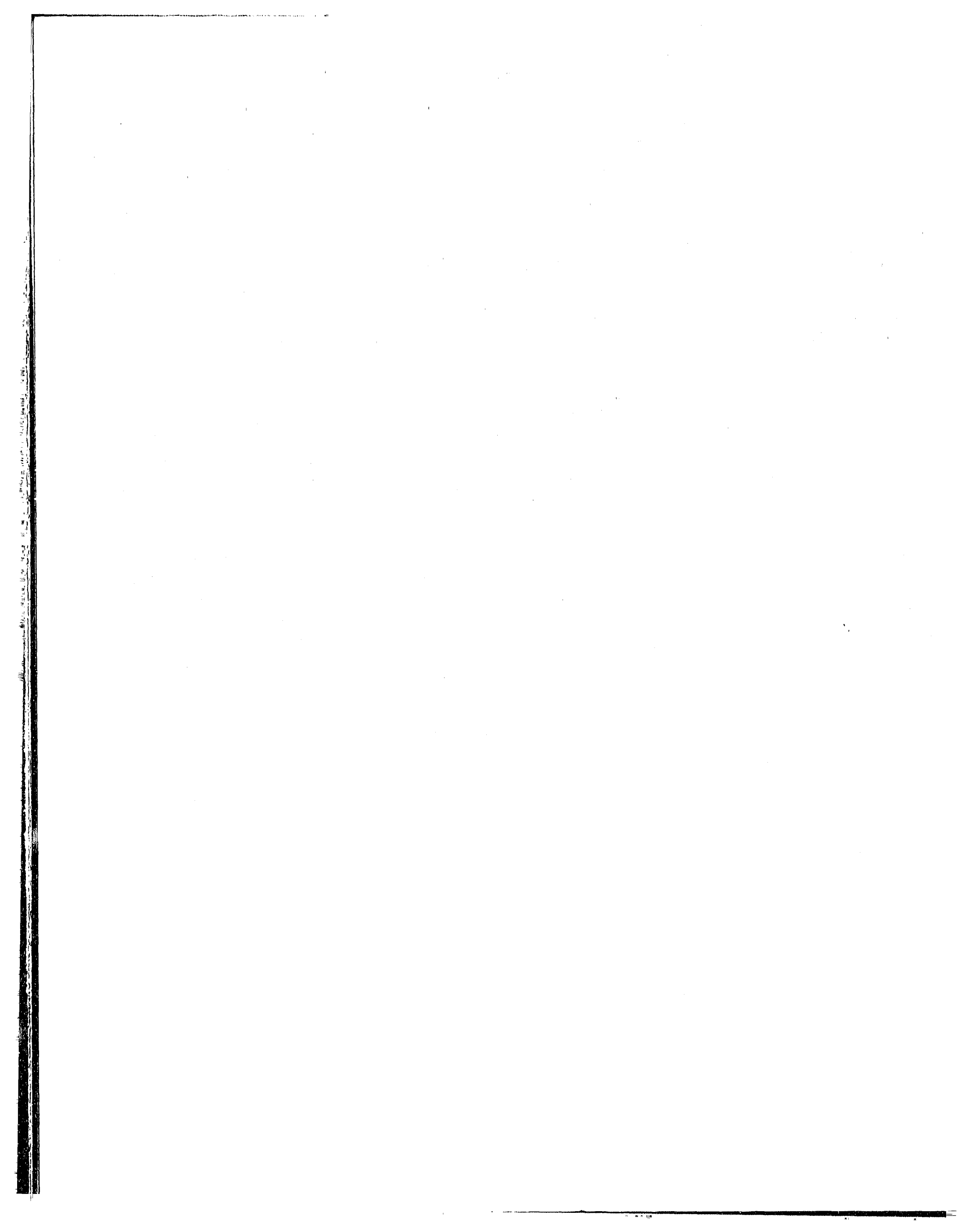

